\title{
Disponibilidad de recursos materiales en el hogar y adquisición de habilidades pre-lectoras
}

\author{
María Laura Andrés \\ Lorena Canet-Juric \\ María M. Richard's \\ Isabel Introzzi \\ Sebastián Urquijo
}

\section{Resumen}

El aprendizaje de la lectura implica un conjunto de factores que hacen posible su adquisición. Entre estos, se destacan el contexto alfabetizador familiar, entendido como el conjunto de los recursos del hogar que abarcan experiencias en las cuales el niño se encuentra en contacto con eventos que involucran la lectoescritura y la disponibilidad de materiales impresos; además de las habilidades y conocimientos prelectores definidos como precursores de la lectura formal, que tienen sus orígenes en las experiencias tempranas de la vida de un niño. El objetivo de este trabajo es explorar y describir las relaciones entre el contexto alfabetizador familiar y el rendimiento en habilidades prelectoras. Para ello se seleccionó una muestra de 88 niños de 5 años de edad, de ambos sexos, y a los cuidadores de los mismos niños a quienes se les administró una entrevista semiestructurada y precodificada denominada Evaluación del Contexto Alfabetizador y la versión en español del Get Ready to Read! Screening Tool de Whitehurst \& Lonigan. Los resultados permitieron establecer una asociación estadísticamente significativa entre los índices disponibilidad de recursos materiales vinculados a la lectura en el hogar -especialmente el acceso a medios tecnológicos- y el rendimiento en habilidades prelectoras, demostrando la importancia que tienen para la alfabetización las características del medio ambiente en el que se desenvuelve el niño.

Palabras clave: Alfabetización, ambiente familiar, materiales instruccionales.

\section{Availability of resources and the acquisiton of pre-reading skills in the family context}

\begin{abstract}
Learning to read involves a number of factors. Among these, we focus on the family context related to literacy. We understand this context as a set of resources, including experiences in which the child is in contact with the events surrounding the literacy and the availability of printed materials, as well as skills and knowledge of pre- reading, identified as precursors of formal reading. In this study we aim to explore and describe the relationships between family background and performance in pre-reading skills. We take, as sample, 88 children 5 years old, both sexes, and their parents. We interviewed the family to evaluate the family context and used the the Spanish version of "Get Ready to Read! Screening Tool" by Whitehurst \& Lonigan. The results indicate a statistically significant association between rates of resource availability of reading materials at home - particularly access to technological means - and performance in pre-reading skills. This reveals the importance of the characteristics of environment in which the child grows to the development of literacy.
\end{abstract}

Keywords: Literacy, home environment, instructional materials

\section{Disponibilidade de recursos materiais e aquisição de habilidades pré-leitoras em contexto familiar}

\section{Resumo}

Aprender a ler envolve um conjunto de fatores. Entre estes, destaca-se a leiturabilidade no contexto da família, entendida como um conjunto de recursos domésticos que incluem experiências em que a criança está em contato com os acontecimentos envolvendo a alfabetização e disponibilidade de materiais; habilidades de pré-leitura e habilidades identificadas como precursoras da leitura formal. O objetivo deste trabalho foi explorar as relações entre os antecedentes familiares e o desempenho de alfabetização em habilidades pré-leitura. Participaram 88 crianças de 5 anos de idade e os seus cuidadores. A eles foram administradas uma entrevista de avaliação de contexto da família e a versão espanhola de Get Ready to Read! Screening Tool. Os resultados indicam uma associação com significância estatística entre as taxas de disponibilidade de recursos materiais de leitura em casa e realização de habilidades pré-leitoras, sugerindo a importância das características do lar para alfabetização.

Palavras-chave: Alfabetização, ambiente familiar, materiais instrucionais. 


\section{Introducción}

El aprendizaje de la lectura implica un conjunto de factores que hacen posible su adquisición eficiente. Entre estos factores se destacan el tipo de método pedagógico empleado por el docente (Hernández \& Jiménez, 1987; Jiménez, Rodrigo, Ortiz, \& Guzmán, 1999), las características de los textos elegidos para la enseñanza (Calero \& Pérez, 1993; Sánchez, 1995), el entrenamiento en los distintos procesos cognitivos lectores (Jiménez e cols., 1999; Ramos Sánchez, 1999), el contexto alfabetizador familiar (Marder, Querejeta, Piacente, Resches, \& Urrutia, 2005) y las habilidades y conocimientos prelectores (Piacente, 2005; Whitehurst \& Lonigan, 2003). Como se puede apreciar, los factores que influyen en el aprendizaje de la lectura constituyen un grupo diverso y heterogéneo. La relación entre las habilidades prelectoras con las que los niños ingresan al sistema escolar y su rendimiento académico posterior en actividades de lectura es considerada significativa (Baydar, Brooks-Gunn, \& Furstenberg, 1993; Stevenson \& Newman, 1986; Tramontana, Hooper, \& Selzer, 1988), por lo que su adquisición temprana resulta fundamental para el aprendizaje inicial de la lectura (Baydar e cols., 1993).

En la adquisición y perfeccionamiento de habilidades de prelectura tienen influencia el contexto alfabetizador familiar en el que se desenvuelve el niño; distintos estudios han encontrado correlaciones significativas entre el contexto alfabetizador familiar y el desarrollo de habilidades lingüísticas y prelectoras en niños de edad preescolar (Bus, van ljzendoorn, \& Pellegrini, 1995; Purcell-Gates, 1996). Dentro del contexto alfabetizador, el nivel socioeconómico de los cuidadores es estimado como un predictor fuerte del desempeño académico de los niños en tareas de lectura y escritura al inicio del primer grado (Magnuson, 2007; Nacional Assessment of Educational Progress, 1991). Asimismo, el nivel educacional de los padres se relaciona positiva y consistentemente con el desempeño escolar (Recart-Herrera, Mathiesen, \& HerreraGarbarini, 2005). También, la participación de los padres en las actividades escolares de sus hijos explica el nivel de los niños en pruebas escolares (Bazán-Ramírez, SánchezHernández, \& Castañeda-Figueiras, 2007).

\section{Contexto Alfabetizador Familiar}

El contexto alfabetizador familiar es un conjunto de recursos que abarca tanto experiencias hogareñas en las cuales el niño se encuentra en contacto con eventos que involucran la lectoescritura como la disponibilidad en su entorno de materiales escritos en diferentes formatos y soportes (Graves, Juel, \& Graves, 2000).

El conjunto de recursos vinculados a las prácticas $y$ creencias alfabetizadoras poseen repercusiones en el desarrollo del vocabulario, de habilidades de prelectura y en el aprendizaje inicial de la lecto-escritura. Aquellas familias en las cuales se da un uso frecuente de actividades de lectura y escritura generan en sus niños conocimientos sobre lo impreso y sobre la funcionalidad y uso del lenguaje escrito
(Purcell-Gates, 1996). Diversos trabajos han aportado evidencia acerca de la asociación significativa entre las expectativas de los padres y el desempeño de los niños al inicio de la alfabetización formal (Halle, Kurtz-Costes, \& Mahoney, 1997). La interacción familiar en lecturas conjuntas tiene un gran impacto sobre el desarrollo de habilidades de prelectura y sobre el aprendizaje de la lectoescritura cuando el niño comienza la escolaridad formal, sobre todo, existe una correlación positiva con el tiempo que destinan los padres a estas prácticas y el grado de compromiso con el que las asumen (Juel, 1991; Braslavsky, 2004).

Aquellos recursos del contexto alfabetizador familiar referidos a la disponibilidad de recursos materiales vinculados a la lectura tienen relación con el nivel cultural, laboral y de ingresos de la familia (Fernández \& Salvador, 1994). El nivel laboral y de ingresos de una familia se ha asociado principalmente con el desempeño académico de niños en edad escolar (Magnuson, 2007; Fueyo, 1990; Gordon \& Greenidge, 1999; Pourtois \& Desmet, 1989). La disponibilidad de medios culturales (libros, periódicos, televisión, enciclopedias, etc.) en el hogar es un componente del contexto alfabetizador que influye en el rendimiento escolar (Echols, West, Stanovich, \& Zehr, 1996). El nivel educativo de los padres influye en la participación de los mismos en las actividades escolares de sus hijos y, como consecuencia, en su rendimiento académico (Bracken \& Fischel, 2008). Se ha encontrado que la privación de estímulos atribuido al déficit sociocultural de diversos entornos, genera diferencias en el rendimiento: en las familias de mayor nivel sociocultural, se ofrece al niño una serie de repertorios educativos mayor que en las de estratos más bajos, lo que permite una mejor adaptación a contextos escolares (Ruiz de Miguel, 1999).

En investigaciones llevadas adelante con niños hispanoparlantes se ha encontrado una enorme variabilidad entre contextos hogareños de diferentes niveles, en relación a la disponibilidad de recursos materiales vinculados a la lectura, y los conocimientos y habilidades infantiles de prelectura que los niños poseen (Dioses e cols., 2006).

\section{Habilidades y conocimientos prelectores}

Las habilidades y conocimientos prelectores hacen referencia a un conjunto de precursores de la lectura formal necesarios para tener éxito en el aprendizaje de la lectura; tienen sus orígenes en las experiencias tempranas de la vida de un niño (Piacente, 2005; Whitehurst \& Lonigan, 2003). Estas habilidades prelectoras se van perfeccionando de forma diferente en cada niño, hasta alcanzar cierto grado de experticia en la lectoescritura (Garton Pratt, 1991). Existen dos áreas específicas que se encuentran en la base del aprendizaje de la lectura y la escritura. Los niños que desarrollan habilidades potentes en esas áreas tienen mayor éxito en el aprendizaje de la lectura. Se trata del conocimiento de lo impreso y la conciencia lingüística.

El conocimiento de lo impreso hace referencia al conocimiento que poseen los niños sobre el material gráfico presente en su entorno cotidiano. Esta habilidad incluye el 
conocimiento de lo escrito y de algunas letras. El conocimiento de lo escrito se refiere al conocimiento general que dispone el niño sobre la escritura como sistema, implicando la comprensión de la funcionalidad de la escritura (Downing, Olilla, \& Oliver, 1975). Es decir, saber que posee y transmite un significado para un otro distante o ausente; conocer la direccionalidad de la escritura y el aspecto visual de un texto. Por último, el conocimiento de las letras se refiere a conocer el grafismo, el sonido de los grafemas o el nombre de algunas letras (Adams, 1990; Compton, 2000; Ferreiro \& Teberosky, 1984; Guardia, 2003; Muter, Hulme, \& Snowling, 1997; Vellutino \& Scanlon, 2002; Whitehurst \& Lonigan, 1998).

Otra de las áreas de habilidades prelectoras extensamente estudiada por su relación con el aprendizaje de la lectoescritura es la conciencia lingüística, se trata de una habilidad metalingüística porque implica la reflexión y el control deliberado sobre el lenguaje (Garton Pratt, 1991). En este dominio, se incluyen las habilidades de conciencia fonológica y de conciencia léxica.

La conciencia fonológica se refiere a la reflexión conciente sobre los sonidos de la propia lengua e implica un conjunto de diferentes habilidades (Defior-Citoler, 1994). Numerosas investigaciones la consideran como la variable con mayor fuerza predictiva de la lectura inicial (Carrillo, 1994; Stanovich \& Siegel, 1994; De Jong \& Van der Leij, 1999; Goswami, 2000; Stanovich, 2000; Vellutino \& Scanlon, 2002). La conciencia fonológica puede ser evaluada a través de tres tareas: comparar sonidos, segmentar en fonemas palabras presentadas como estímulo y unir fonemas para formar palabras (Torgesen \& Mathes, 2002).

La conciencia léxica se trata de la capacidad para identificar una palabra como un elemento lingüístico separado del continuo del habla. Esta habilidad puede evaluarse a través de tareas en las que el niño debe aislar las palabras que componen una oración y reconocer qué palabras cortas conforman otra palabra más larga (Borzone, Rosemberg, Diuk, Silvestre, \& Plana, 2004).

En síntesis, las habilidades y conocimientos prelectores constituyen un conjunto heterogéneo que permitirán al niño los primeros acercamientos con las formas propias de la lectura y la escritura y serán estos primeros contactos los que facilitarán los aprendizajes esenciales de la lectoescritura, a su vez, estas habilidades se adquieren en la interacción del niño con contextos alfabetizadores que favorezcan el acceso a las múltiples formas de la cultura escrita.

Teniendo en cuenta que escasas investigaciones en niños hispanoparlantes han explorado las relaciones entre el contexto alfabetizador familiar y la adquisición de habilidades prelectoras, el objetivo de este trabajo fue explorar y describir estas relaciones en un grupo de niños de 5 años de edad de Jardín de Infantes sosteniendo la hipótesis principal de que el contexto alfabetizador familiar guarda relación con el rendimiento diferencial de las habilidades prelectoras de los niños.

\section{Método}

\section{Participantes}

Se seleccionó de forma no probabilística casual una muestra de 88 niños y niñas de 5 años de edad, alumnos de sala de 5 del Jardín de Infantes de escuelas de gestión privada de la ciudad de Mar del Plata, Argentina. Se entrevistó personalmente a los cuidadores o tutores legales de los niños evaluados (en su mayoría fueron los padres o familiares directos). La muestra de niños estaba conformada por $53,4 \%$ de mujeres y $46,6 \%$ de varones. De los cuidadores que participaron de la entrevista, el $89,7 \%$ fueron las madres y el $10,3 \%$ los padres de los niños. Todos informaron saber leer y escribir.

\section{Instrumentos}

Para la evaluación del contexto alfabetizador familiar se utilizó una entrevista semiestructurada y precodificada de elección múltiple (3 a 6 opciones), denominada Evaluación del Contexto Alfabetizador (Piacente, Querejeta, Marder, \& Resches, 2003). La misma fue construida para éste y otros estudios de similar naturaleza (Piacente, Marder, Resches, \& Ledesma, 2006). Este instrumento fue elaborado a partir de la 'Stony Brook Family Reading Survey' (Whitehurst, 1992) y de la 'Stony Brook Family Survey for Elementary School' (Whitehurst, 1997). Esta entrevista evalúa diferentes dimensiones del contexto alfabetizador familiar; cada dimensión o sub-escala se conforma de 7 ítems con respuestas en formato escala Lickert en su mayoría. Las sub-escalas que evalúan distintas dimensiones son: (1) Disponibilidad de recursos materiales vinculados a la lectura: indaga la existencia y cantidad de libros en general, de libros específicos para los niños y de papel y lápiz con los que se cuenta en el hogar. Adicionalmente, se pregunta por la cantidad de personas que viven en la casa y por el idioma de la familia. Su importancia reside en que constituyen elementos de suma importancia para las interacciones con el lenguaje escrito. (2) Características de hábitos y comportamientos del niño vinculados al lenguaje escrito: indaga sobre aspectos referidos a frecuencia de actividades de lectura y escritura de parte del niño, disfrute de las actividades de lectura compartidas, preguntas referidas a letras y palabras y escritura del nombre propio y otras palabras. (3) Nivel educativo de los padres o cuidadores: explora años de escolaridad aprobados. Adicionalmente, indagaba por el tipo de ocupación del cuidador. (4) Creencias y prácticas alfabetizadoras: se refiere a frecuencia e importancia concedida a actividades tales como cantar canciones y contar cuentos, enseñanza explícita de letras y números, ayuda en la preparación del material escolar.

Para la evaluación de las habilidades prelectoras se utilizó la versión en español del Get Ready to Read! Screening Tool (Whitehurst \& Lonigan, 2003). El test se compone de 20 ítems en donde el niño debe señalar dentro de un con- 
junto de 4 posibilidades presentadas en forma gráfica aquella correcta según la consigna del evaluador, de los cuales 10 ítems corresponden a conocimiento de lo impreso y 10 a conciencia lingüística. Específicamente 5 ítems evalúan conocimiento de lo escrito a través de consignas como 'Algunos niños escribieron cuentos. Encuentra el más largo'; otros 5 ítems evalúan conocimiento de letras, con consignas tales como 'Encuentra el dibujo que tiene letras'. Para conciencia fonológica se presentan 5 ítems con instrucciones como 'Esta es una bola, y estos dibujos son: un caballo, un zapato, una pared y una ola. Encuentra el que rima con bola' y para conciencia léxica se utilizan 5 ítems con consignas tales como 'Estos dibujos son: un girasol, una raqueta, un zapato y una chimenea. Encuentra lo que obtienes cuando pones GIRA y SOL juntos'. La prueba se puntúa 1 si la respuesta señalada es la correcta y 0 si se señala una respuesta incorrecta. El puntaje máximo posible es de 20 puntos. El instrumento proporciona información sobre distintos niveles de habilidades prelectoras utilizando para ello la sumatoria de los ítems correctamente respondidos a lo largo de la prueba, más allá del tipo de habilidad a la cual pertenezcan. Los distintos niveles refieren a: Nivel 1: escasas habilidades (de 0 a 4 habilidades). Nivel 2: habilidades que están comenzando a desarrollarse (de 5 a 8 habilidades). Nivel 3: habilidades que indican un mayor progreso (de 9 a 12 habilidades). Nivel 4: habilidades que indican una preparación medianamente elevada para la lectura (de 13 a 16 habilidades). Nivel 5: habilidades que indican una preparación suficientemente elevada para la lectura (de 17 a 20 habilidades). La confiabilidad de la prueba de habilidades prelectoras según la fórmula de Spearman-Brown de bipartición fue =.69. El alfa de Cronbach fue .73 .

\section{Procedimientos}

Se pidió a los cuidadores un consentimiento por escrito para permitir la participación de sus hijos en la investigación. Se aplicó la evaluación de habilidades prelectoras cuando se encontraban al inicio del segundo semestre de la sala de 5 del Jardín de Infantes en un aula de una institución destinada para tal fin. Se concertó previamente con los cuidadores un horario de reunión y se les administró de forma individual la entrevista sobre contexto alfabetizador familiar en un aula de la escuela determinada.

\section{Análisis estadístico}

Para el análisis de los resultados se utilizaron los recursos de la estadística descriptiva e inferencial. Para facilitar la interpretación de los datos en función de los objetivos e hipótesis planteados, se construyeron cuatro índices correspondientes a las dimensiones de la Evaluación del Contexto Alfabetizador. Para ello, se categorizaron los ítems otorgándoles puntajes que variaban entre 1: escaso, 2: intermedio y 3: adecuado. Los puntajes de los ítems fueron sumados y luego divididos por la cantidad total de ítems para que fueran equivalentes, ya que el número de ítems que integran el índice no eran idénticos para todos los casos. Los índices que quedaron conformados fueron: (1) Índice Disponibilidad de Recursos (IDR), se denominó así al conjunto de ítems que describían la disponibilidad de recursos materiales vinculados a la lectura. (2) Índice Hábitos del niño en lectura (ILN), incluía características de hábitos y comportamientos del niño vinculados al lenguaje escrito. (3) Índice Nivel Educativo de los Padres (IEP), estaba conformado por aquellos ítems que describían el nivel educativo de padres y finalmente el (4) Índice Creencias y Prácticas alfabetizadoras (ICP), que evaluaba tipo de creencias y frecuencia de prácticas alfabetizadoras en el hogar del niño. Se aplicó prueba de independencia Chi cuadrado para medir la asociación con el rendimiento de habilidades prelectoras. Se realizó un Análisis Factorial Exploratorio del IDR, que es el que presentó asociaciones estadísticamente significativas con las habilidades prelectoras. Se analizaron las relaciones entre los niveles de disponibilidad de recursos materiales vinculados a la lectura y los rendimientos de habilidades prelectoras. Para ello, se dividió la muestra en tres grupos de acuerdo a la puntuación en el IDR, considerándose por debajo de un desvío estándar (-1 $D E)$ Baja Disponibilidad de Recursos, por arriba de un desvío estándar $(+1 D E)$ se consideró Alta Disponibilidad y para aquellas puntuaciones ubicadas en el centro (-1 DE y +1 DE) Disponibilidad Media. Se aplicó la Prueba no paramétrica de diferencia de medias de Kruskal-Wallis (Kolmogorov-Smirnov $(91)=160, p<0.001)$.

\section{Resultados}

En relación al contexto alfabetizador familiar, respecto a la disponibilidad de recursos materiales vinculados a la lectura, los resultados de las encuestas indicaron que el $43 \%$ de los hogares presentaron más de 40 libros de lectura, que el $54 \%$ disponía tanto de televisor como de computadora y que en el $38,6 \%$ de los hogares se le permitía al niño utilizar la computadora. Sobre las características de hábitos y comportamientos del niño vinculados a la lectura, casi la totalidad de los niños $(96,6 \%)$ no presentaba dificultades de comprensión oral, solicitaban a los adultos del entorno que les lean libros de cuentos u otros materiales $(86,1 \%)$ y hojeaban libros y revistas por su cuenta dos o más veces a la semana $(95,4 \%)$. En relación al nivel educativo de los cuidadores, el $64,8 \%$ de los informantes poseían entre 10 y 15 años de escolaridad, un $20,5 \%$ informó ser profesional, un $4,5 \%$ técnico, un $23,9 \%$ empleado de comercio, el 5,7\% poseía un oficio, el $37,5 \%$ de los informantes eran amas de casa y el $8 \%$ restante se encontraba desempleado o prefirió no contestar respecto de su ocupación. Con relación a las creencias y prácticas alfabetizadoras, el $65,9 \%$ de los padres indicó que enseñaban letras y números a sus hijos y el $66,7 \%$ a escribir su nombre y otras palabras de uso cotidiano con una frecuencia de más de dos veces por semana.

En cuanto a las habilidades prelectoras, el $45,5 \%$ de los niños se encontraban en el Nivel 5, que implica una preparación suficientemente elevada como para comenzar el aprendizaje de la lectura. 
Tabla 1. Niveles de habilidades prelectoras para los niños en sala de 5 del Jardín de Infantes.

\begin{tabular}{lcccc}
\hline & Frecuencia & Porcentaje & $\begin{array}{c}\text { Porcentaje } \\
\text { acumulado }\end{array}$ \\
\hline Válidos & Nivel 3 (Bajo) & 10 & 11,4 & 11,4 \\
\hline Nivel 4 (Medio) & 38 & 43,2 & 54,5 \\
\hline Nivel 5 (Alto) & 40 & 45,5 & 100,0 \\
\hline Total & 88 & 100,0 & \\
\hline
\end{tabular}

Tabla 2. Asociación entre los Índices de Contexto Alfabetizador y las Habilidades Prelectoras según prueba de independencia de chi-cuadrado.

Habilidades Prelectoras

\begin{tabular}{|c|c|c|}
\hline \multirow{3}{*}{$\begin{array}{l}\text { Índice Disponibilidad de Recursos } \\
\text { (IDR) }\end{array}$} & Chi-cuadrado & 11,659 \\
\hline & $\mathrm{GI}$ & 4 \\
\hline & Sig. &, $020\left(^{*}\right)$ \\
\hline \multirow{3}{*}{$\begin{array}{l}\text { Índice Hábitos del niño en Lectura } \\
\text { (ILN) }\end{array}$} & Chi-cuadrado & 4,524 \\
\hline & $\mathrm{Gl}$ & 4 \\
\hline & Sig. &, $340(a)$ \\
\hline \multirow{3}{*}{$\begin{array}{l}\text { Índice Nivel Educativo de los } \\
\text { Padres (IEP) }\end{array}$} & Chi-cuadrado & 7,733 \\
\hline & $\mathrm{GI}$ & 6 \\
\hline & Sig. & ,258(a) \\
\hline \multirow{3}{*}{$\begin{array}{l}\text { Índice Creencias y Prácticas } \\
\text { Alfabetizadoras (ICP) }\end{array}$} & Chi-cuadrado & 3,877 \\
\hline & $\mathrm{GI}$ & 6 \\
\hline & Sig. & ,693(a) \\
\hline
\end{tabular}

\section{Relaciones entre los índices del contexto alfabetizador familiar y las habilidades prelectoras}

De los cuatro índices construidos, solamente uno de ellos -el Índice Disponibilidad de recursos materiales (IDR)aparece asociado de forma estadísticamente significativa con los niveles de habilidades prelectoras. En el IDR, las diferencias entre los niveles de habilidades prelectoras fueron estadísticamente significativas $\chi^{2}(4,88)=11.65, p=0.02$, observándose una asociación directamente proporcional, es decir que a mayores valores del IDR mayores niveles en las habilidades prelectoras. La fuerza de esta asociación resulta moderada ( $C$ de Pearson=0.347). La lectura de perfiles de la tabla de contingencia nos indica que del $100 \%$ de los niños con valores bajos en el IDR, solamente el $5.9 \%$ posee el nivel más alto en las habilidades prelectoras, en tanto que del $100 \%$ de los alumnos con valores altos en el mismo IDR ese porcentaje es de $30.6 \%$.

A continuación, se presentan los resultados del Análisis Factorial Exploratorio (AFE) del IDR con el objeto de evaluar los componentes de la estructura factorial del mismo que, como se ha mencionado, es el único que mostró una asociación estadísticamente significativa con los niveles de rendimiento en habilidades prelectoras. 
Tabla 3. Análisis Factorial Exploratorio de los ítems del Índice Disponibilidad de Recursos Materiales (IDR). Método de extracción Factorización Alfa. Varianza total explicada de los 7 ítems del IDR para la muestra total.

\begin{tabular}{lrrr}
\hline \multirow{2}{*}{ Factor } & \multicolumn{3}{c}{ Autovalores iniciales } \\
\cline { 2 - 4 } & \multicolumn{2}{c}{ Total } & \multicolumn{2}{c}{ \% de la varianza } & \% acumulado \\
\hline 1 & 2,745 & 39,211 & 39,211 \\
\hline 2 & 1,254 & 17,915 & 57,126 \\
\hline 3 & 1,134 & 16,196 & 73,322 \\
\hline 4 &, 831 & 11,874 & 85,196 \\
\hline 5 &, 684 & 9,775 & 94,970 \\
\hline 6 &, 211 & 3,017 & 97,987 \\
\hline 7 &, 141 & 2,013 & 100,000 \\
\hline
\end{tabular}

Tabla 4. Comunalidades por Factor del Análisis Factorial Exploratorio de los ítems del Índice de Disponibilidad de Recursos Materiales (IDR). Método de extracción Factorización Alfa.

\begin{tabular}{llll}
\hline \multicolumn{1}{c}{ Ítem } & Factor I & Factor II & Factor III \\
\hline 1. Quiénes viven en la casa &,- 495 &,- 015 &,- 600 \\
\hline 2. Idioma &,- 163 &,- 002 &, 837 \\
\hline 3. Cantidad de libros &, 358 &, 768 &,- 176 \\
\hline 4. Cantidad de libros para niños &, 303 &, 841 &,- 088 \\
\hline 5. Cantidad de materiales y útiles escolares &,- 142 &, 670 &,- 034 \\
\hline 6. Presencia de televisor y/o computadora &, 843 &, 180 &,- 021 \\
\hline 7. Niño como usuario de la computadora &, 896 &, 136 &,- 037 \\
\hline
\end{tabular}

La medida de adecuación muestral de Kaiser-MeyerOlkin (KMO) evalúa la dependencia de las variables; como se observa que el valor se aproxima a 1 (0.612) entonces tiene sentido realizar el análisis factorial. Por otro lado, la Prueba de esfericidad de Bartlett indica que se contrasta la hipótesis nula de que las matrices de correlaciones poblacionales son iguales a la matriz de identidad, lo que muestra que las variables se relacionan $\left[\chi^{2}(21,88)=216.95, p=\leq .01\right]$.

El análisis realizado permite identificar tres factores con autovalores superiores a 1 que explican el $73.32 \%$ de la varianza. Los factores I (Disponibilidad de medios tecnológicos) y II (Disponibilidad de libros y materiales escolares) son los que explican el mayor porcentaje de varianza y corresponden a la disponibilidad y utilización de los medios tecnológicos presentes en el hogar y a la disponibilidad de materiales escolares didácticos y literarios.

\section{Niveles de disponibilidad de recursos y rendimiento en habilidades prelectoras}

Para evaluar el rendimiento diferencial en habilidades prelectoras según los niveles de disponibilidad de recursos materiales vinculados a la lectura, se aplicó la prueba de Kruskal-Wallis. Los resultados se presentan en la tabla 5. 
Tabla 5. Prueba de Kruskal- Wallis para constrastes de medias de rendimiento en habilidades prelectoras y niveles de disponibilidad de recursos.

\begin{tabular}{llrr}
\hline & Índice Disponibilidad de & N & \multicolumn{1}{c}{$\begin{array}{c}\text { Rango } \\
\text { promedio }\end{array}$} \\
\hline Habilidades prelectoras & Recursos Materiales (IDR) & 17 & 26,29 \\
\cline { 2 - 4 } & Daja Disponibilidad & 39 & 45,13 \\
\cline { 2 - 4 } & Alta Disponibilidad & 29 & 49,93 \\
\cline { 2 - 4 } & Total & 85 & \\
\hline Chi-cuadrado & Habilidades prelectoras & \\
\hline Gl & & 10,478 & \\
\hline Sig. asintót. & & 2 & \\
\hline
\end{tabular}

a Prueba de Kruskal-Wallis

b Variable de agrupación: Índice Disponibilidad de Recursos Materiales (IDR)

Tabla 6. Estadísticos para la prueba $U$ de Mann-Whitney de contrastes entre medias según nivel de disponibilidad de recursos.

\begin{tabular}{|c|c|c|c|c|}
\hline & $\begin{array}{l}\text { Índice Disponibilidad de } \\
\text { Recursos Materiales (IDR) }\end{array}$ & $\mathbf{N}$ & $\begin{array}{c}\text { Rango } \\
\text { promedio }\end{array}$ & $\begin{array}{l}\text { Suma de } \\
\text { rangos }\end{array}$ \\
\hline \multirow{4}{*}{ Habilidades prelectoras } & Baja Disponibilidad & 17 & 14,97 & 254,50 \\
\hline & Alta Disponibilidad & 29 & 28,50 & 826,50 \\
\hline & Total & 46 & & \\
\hline & & \multicolumn{3}{|c|}{ Habilidades prelectoras } \\
\hline U de Mann-Whitney & & \multicolumn{3}{|c|}{101,500} \\
\hline W de Wilcoxon & & \multicolumn{3}{|c|}{254,500} \\
\hline Z & & \multicolumn{3}{|c|}{$-3,333$} \\
\hline Sig. asintót. (bilateral) & & \multicolumn{3}{|c|}{,001 } \\
\hline
\end{tabular}

a Variable de agrupación: Índice Disponibilidad de Recursos Materiales (IDR)

Los resultados de los estadísticos de contraste muestran que existen diferencias estadísticamente significativas en los rendimientos de habilidades prelectoras para los distintos niveles de disponibilidad de recursos materiales $\left(\chi^{2}\right.$ $(2 \mathrm{gl})=10.478 ; p<.05)$.
Para analizar qué grupos presentan diferencias entre sí se aplicó la prueba $U$ de Mann-Whitney para dos muestras independientes. Se utilizó la corrección de Bonferroni para controlar la tasa de errores tipo I $(p<.017)$, la diferencia resultó significativa entre los niveles bajo y alto $(U=101,5 ; p<.017)$. 


\section{Discusión}

En este estudio nos propusimos evaluar la asociación entre el contexto alfabetizador familiar y el rendimiento en habilidades de prelectura, considerando que otras investigaciones han encontrado relaciones significativas entre estas variables (Bus e cols., 1995; Purcell-Gates, 1996). En este trabajo, los resultados permitieron confirmar tan sólo la existencia de asociaciones entre la disponibilidad de recursos materiales vinculados a la lectura en el hogar y el rendimiento en habilidades prelectoras.

Nuestros resultados están en consonancia con trabajos que plantean que la disponibilidad de medios culturales -que comprende material escolar, material de lectura (en particular libros para niños) y medios tecnológicos- es un componente del contexto alfabetizador que posee influencia en el rendimiento escolar (Echols e cols., 1996; Bowey, 1995). Dentro de estos recursos, el que más se destaca en la asociación con el rendimiento en habilidades prelectoras, es el acceso a medios tecnológicos, como la computadora y la televisión. Bussière y Gluszynki (2004) han reportado la existencia de correlaciones significativas entre el uso de la computadora y el rendimiento en lectura. En este sentido, es posible pensar al medio tecnológico como facilitador de numerosos estímulos escritos que propician la familiarización del niño con la escritura. Es evidente que la computadora es un instrumento tecnológico portador de textos escritos, de mayor valor motivacional que un libro en función de la inclusión de imágenes, animaciones, etc.

No obstante, no todos los niños poseen el mismo nivel de acceso a estos medios. Evidentemente diferentes niveles de exposición a materiales en el hogar, ofrecen distintas oportunidades para el aprendizaje y, consecuentemente, se reflejan en el rendimiento escolar. Existen estudios que afirman que tanto la disponibilidad de material escrito como la cantidad de libros en el hogar, poseen efectos significativos en la adquisición de nuevo vocabulario y de habilidades de prelectura en niños de edad preescolar (Echols e cols., 1996). Es decir, que para la adquisición de habilidades de prelectura, los niños necesitan diversos materiales; el hecho de disponer de libros, papeles, herramientas de escritura, etc. en su medio cotidiano los predispone a prácticas de lectura y de escritura (Slegers, 1996).

En síntesis, la adquisición temprana de habilidades de prelectura repercute favorablemente en el aprendizaje de la lectoescritura (Baydar e cols., 1993; Stevenson \& Newman, 1986; Tramontana e cols., 1988). Estas habilidades se desarrollan en el contexto alfabetizador familiar (Purcell-Gates, 1996). La disponibilidad de recursos materiales vinculados a la lectura, que son fundamentales en este contexto, propicia la exposición de los niños a experiencias tempranas que amplían su mundo inmediato, lo que se deriva, por lo general, en un mejor desempeño escolar (Raz \& Bryant, 1990; Whitehurst \& Lonigan, 1998).

Por lo tanto, los resultados de este estudio nos permiten sostener la idea de la necesidad de disminuir la brecha tecnológica que actualmente diferencia a los hogares de niveles socio económicos y culturales bajos de los altos. El acceso a la tecnología se relaciona directamente a las posibilidades de aprendizaje de la lectoescritura y, sin duda, al desempeño académico de los niños. Por lo tanto, debemos insistir en el valor que tiene la tecnologización de las escuelas de gestión pública que atienden a los sectores más desfavorecidos de nuestra sociedad y que se caracterizan por el escaso acceso a los medios tecnológicos que tienen impacto directo sobre su educación.

\section{Referências}

Adams, M. J. (1990). Learning to read: thinking and learning about print. Cambridge, MA: MIT Press.

Baydar, N., Brooks-Gunn, J., \& Furstenberg, F. F. (1993). Early warning signs of functional illiteracy: Predictors in childhood and adolescence. Child Development, 64, 815-829.

Bazán-Ramírez, A., Sánchez-Hernández, B. A., \& CastañedaFigueiras, S. (2007). Relación estructural entre el apoyo familiar, nivel educativo de los padres, características del maestro y desempeño en lengua escrita. Revista Mexicana de Investigación Educativa, 12(33), 701-729.

Borzone, A. M, Rosemberg, C. R., Diuk, B., Silvestre, A., \& Plana, D. (2004) Niños y maestros por el camino de la alfabetización. Buenos Aires: Red de Apoyo Escolar.

Bowey, J. A. (1995). Socieconomic status differences in preschool phonological sensitivity amd first-grade reading achievement. Journal of Educational Psychology, 87, 476-487.

Bracken, S. S., \& Fischel, J. E. (2008). Family reading behavior and early literacy skills in preschool children from low-income backgrounds. Early Education and Development, 19(1), 45-67.

Braslavsky, B. (2004). ¿Primeras Letras o Primeras Lecturas? Una Introducción a la Alfabetización Temprana. Buenos Aires: Fondo de Cultura Económica.

Bus, A. G., van IJzendoorn, M. H., \& Pellegrini, A. (1995). Joint book reading makes for success in learning to read: $A$ meta-analysis on intergeneracional transmisión of literacy. Review of Educational Research, 65, 1-21.

Bussiére, P., \& Gluszynski, T. (2004) The impact of Computer Use on Reading Achievement of 15-year-olds. Final Report. Québec: Learning Policy Directorate, Strategic Policy and Planning Branch.

Calero, A., \& Pérez, R. (1993). Segmentación del habla y adquisición de la lectura. Comunicación, Lenguaje y Educación, 18, 41-53.

Carrillo, M. S. (1994). Developmental of phonological awareness and reading acquisition. Reading and Writing: An Interdisciplinary Journal, 6, 279-298. 
Compton, D. (2000). Modelling the growth of decoding skills in first grade children. Scientific Studies of Reading, 4, 219-259.

De Jong, P. F., \& Van der Leij, A. (1999). Specific contributions of phonological habilities to early reading acquisition: Results from a Dutch latent variable longitudinal study. Journal of Educational Psychology, 91(3), 450-476.

Defior-Citoler, S. A. (1994). La consciencia fonológica y la adquisición de la lectoescritura. Infancia y Aprendizaje, 67-68, 91-113.

Dioses, A. S., Lupe-García, A., Matalinares C., M., Abel Cuzcano, Z., Pancaposte, N. Quiroz, J. W. e cols. (2006). Análisis Psicolingüístico del desarrollo fonético-fonológico de alumnos preescolares de Lima Metropolitana. Revista de Investigación Psicológica, 9(2): 9-32.

Downing, J., Olilla, L., \& Oliver, P. (1975). Cultural differences in children's concepts of reading and writing. Educational Psychology, 45, 312-316.

Echols, L. D., West, R. F., Stanovich, K. E., \& Zehr, K. S. (1996). Using children's literacy activities to predict growth in verbal cognitive skills: A longitudinal investigation. Journal of Educational Psychology, 88, 296-304.

Fernández, S., \& Salvador, F. (1994). La familia ante el fracaso escolar. Educadores, 36(169), 7-22.

Ferreiro, E., \& Teberosky, A. (1984). Los sistemas de escritura en el niño. México: Siglo XXI.

Fueyo, A. (1990). El fracaso escolar: entre la ideología y la impotencia. Educadores, 153, 25-40.

Garton, A., \& Pratt, C. (1991). Aprendizaje y proceso de alfabetización. El desarrollo del lenguaje hablado y escrito. Barcelona: Paidós.

Gordon, J., \& Greenidge, J. (1999). Europa: El fracaso escolar y sus consecuencias. Organización y Gestión Educativa, 3, 4-8.

Goswami, U. (2000). Phonological representations, reading development and dyslexia: towards a cross-linguistic theoretical framework. Dislexia, 6(2): 133-151.

Graves, M., Juel, C., \& Graves, B. (2000). Teaching reading in the 21st century (3a ed.). Needham Heights, MA: Allyn \& Bacon.

Guardia, P. (2003). Relaciones entre habilidades de alfabetización emergente y la lectura desde el nivel de transición mayor a primero básico. Psykhé, 12, 63-79.

Halle, T., Kurtz-Costes, B., \& Mahoney, J. (1997). Family influences on school achievement in low income, Africam american children. Journal of Educational Psychology, 89, 527-537.
Hernández, P., \& Jiménez, J. (1987). Influencia de los métodos de lectura en los hábitos de trabajo intelectual de los escolares. Infancia y Aprendizaje, 38, 15-26.

Jimenez, J. E., Rodrigo, M., Ortiz, M. R., \& Guzmán, R. (1999). Dossier Documental. Procedimientos de Evaluación e Intervención en el aprendizaje de la lectura y sus dificultades desde una perspectiva cognitiva. Infancia y Aprendizaje, 88, 107-122.

Juel, C. (1991). Beginning Reading. Em R. Barr, M. L. Kamil, P. B Mosenthal \& P. D. Pearson (Eds.), Handbook of Reading Research (Volume II) (pp. 759-789). Mahwah, NJ: Lawrence Erlbaum Associates.

Magnuson, K. (2007). Maternal Education and Children's Academic Achievement during middle childhood. Developmental Psychology, 43(6), 1497-1512.

Marder, S., Querejeta, M., Piacente, T., Resches, M., \& Urrutia, M. I. (2005). Características del contexto alfabetizador en familias de diferente nivel socioeconómico. Un estudio comparativo. Em E. Diez-Villoria, B. Zubiauz \& M. A. Mayor Cinca (Eds.), Estudio sobre la Adquisición del Lenguaje (pp. 803-818). Salamanca: Ediciones Universidad de Salamanca.

Muter, V., Hulme, C., \& Snowling, M. (1997). Segmentation, not rhyming, predicts early progress in learning to read. Journal of Experimetal Child Psichology, 63, 370-396.

National Assessment of Educational Progress. (1991). The 198990 national assessment of reading and literature. Denver, CO: Author.

Piacente, T. (2005). Las habilidades y conocimientos prelectores, su incidencia en el aprendizaje formal. Em J. Vivas (Ed.), Las Ciencias del Comportamiento en los albores del Siglo XXI. XRAACC. Mar del Plata: UNMDP.

Piacente, T., Querejeta, M., Marder, S., \& Resches, M. (2003). Evaluación del Contexto Alfabetizador. La Plata, Comisión de Investigaciones Científicas (Inédito. Mimeo).

Piacente, T., Marder, S., Resches, M., \& Ledesma, R. (2006) El contexto alfabetizador hogareño en familias de la pobreza. Comparación de sus características con las de familias no pobres. RIDEP, 1(21), 61-88.

Pourtois, J. P., \& Desmet, H. (1989). L'education familial. Revue Francaise de Pedagogie, 86, 69-101.

Purcell-Gates, V. (1996). Stories, coupons, and the TV Guide: Relationship between home literacy experiences and emergent literacy knowledge. Reading Research Quarterly, 31, 406-428.

Ramos Sánchez, J. L. (1999). Una perspectiva cognitiva de las dificultades lectoescritoras. Procesos de evaluación e intervención. Madrid: Ciudad Nueva. 
Raz, I. S., \& Bryant, P. (1990). Social background, phonological awareness and children's reading. British Journal of Developmental Psychology, 8, 209-225.

Recart-Herrera, M. I., Mathiesen-De Gregori, M. E., \& HerreraGarbarini, M. O. (2005). Familia del Preescolar y su Desempeño Escolar Posterior. Revista Enfoques Educacionales, 7(1), 105123.

Ruiz de Miguel, C. (1999). La familia y su implicación en el desarrollo infantil. Revista Complutense de Educación, 10(I), 289-304.

Sánchez, M. (1995). Los Textos Expositivos. Estrategias para mejorar su comprensión. Buenos Aires: Santillana.

Slegers, B. (1996) A review of the research and literature on emergent literacy. Urbana Champaing IL: ERIC Clearinghouse on Elementary and Early Childhood Education (ERIC Document Reproduction Service No. ED 397 959).

Stanovich, K. E. (2000) Progress in Understanding Reading. Scientific Foundationes and New Frontiers. New York: The Guilford Press.

Stanovich, K. E., \& Siegel, L. (1994). The phenotypic performance of reading disables children: a regresión based test of the phonological-core variable difference model. Journal of Educational Psychology, 86, 221-234.

Stevenson, H. W., \& Newman, R. S. (1986). Long-term prediction of achievement and attitudes in mathematics and reading. Child Development, 57, 646-659.
Torgesen, J. K., \& Mathes, P. G. (2000). A Basic Guide to Understand, Assessing, and Teaching Phonological Awareness. Austin, Texas: Pro-De International Publisher.

Tramontana, M. G., Hooper, S., \& Selzer, S. C. (1988). Research on preschool prediction of later academia achievment: A review. Developmental Review, 8, 89-146.

Vellutino, F. R., \& Scanlon, D. M. (2002). Emergency literacy skills, early instruction and individual differences as determinants or difficulties in learning to read: The case for early intervention. En S. Neuman \& D. Dickinson (Eds.), Handbook of early literacy research (pp. 295-321). Londres: The Guildford Press.

Whitehurst, R. (1992) Stony Brook Family Reading Survey. Stony Brook, NY: Published by the author.

Whitehurst, R. (1997) Stony Brook Family Survey for Elementary School. Stony Brook, NY: Published by the author.

Whitehurst, R., \& Lonigan, C. J. (1998). Child development and emergent literacy. Child Development, 69, 848-872.

Whitehurst, G. J., \& Lonigan, C. (2003). Get Teady to read! An Early Literacy Manual: Screening Tool, Activities and Resources. USA: Pearson Early Learning.

\section{Sobre os autores}

María Laura Andrés (mlandres@mdp.edu.ar)

Facultad de Psicología, Universidad Nacional de Mar del Plata, Mar Del Plata, Argentina

Lorena Canet-Juric (Iorenacanet@yahoo.com.ar)

Facultad de Psicología, Universidad Nacional de Mar del Plata, Mar Del Plata, Argentina

María M. Richard's (mmrichar@mdp.edu.ar)

Facultad de Psicología, Universidad Nacional de Mar del Plata, Mar Del Plata, Argentina

Isabel Introzzi (isaintrozzi@hotmail.com)

Facultad de Psicología, Universidad Nacional de Mar del Plata, Mar Del Plata, Argentina

Sebastián Urquijo (urquijo@mdp.edu.ar)

Facultad de Psicología, Universidad Nacional de Mar del Plata, Mar Del Plata, Argentina

\section{Correspondência}

María Laura Andrés

Universidad Nacional de Mar del Plata, Facultad de Psicología, Dean Funes 3350, Cuerpo V, Nivel III (7600), Mar del Plata, Argentina. 